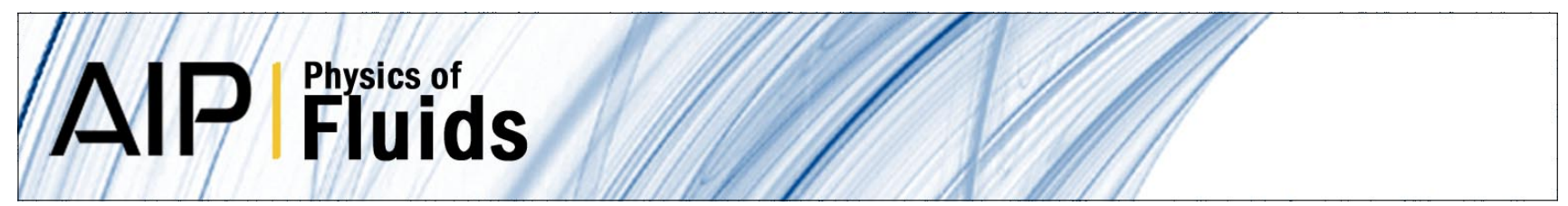

\title{
Transport processes in random arrays of cylinders. II. Viscous flow
}

\author{
A. S. Sangani and C. Yao
}

Citation: Phys. Fluids 31, 2435 (1988); doi: 10.1063/1.866596

View online: http://dx.doi.org/10.1063/1.866596

View Table of Contents: http://pof.aip.org/resource/1/PFLDAS/v31/i9

Published by the American Institute of Physics.

\section{Related Articles}

Note: Temperature derivative of the refractive index of binary mixtures measured by using a new thermodiffusion cell

Rev. Sci. Instrum. 82, 126105 (2011)

Can convection induced by heating delay a thermal explosion?

Phys. Fluids 20, 104107 (2008)

Limitations of linear control of thermal convection in a porous medium

Phys. Fluids 18, 074109 (2006)

Shock velocity and temperature measurements of plastic foams compressed by smoothed laser beams Phys. Plasmas 12, 012706 (2005)

How ions distribute in a drying porous medium: A simple model

Phys. Fluids 14, 1389 (2002)

\section{Additional information on Phys. Fluids}

Journal Homepage: http://pof.aip.org/

Journal Information: http://pof.aip.org/about/about_the_journal

Top downloads: http://pof.aip.org/features/most_downloaded

Information for Authors: http://pof.aip.org/authors

\section{ADVERTISEMENT}

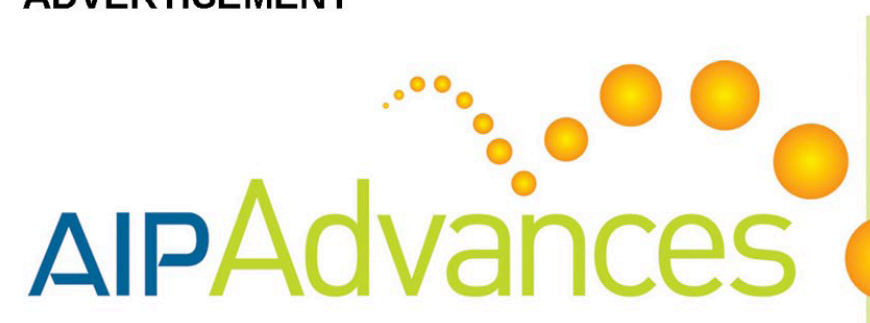

Explore AIP's new open-access journal

Article-level metrics now available

Join the conversation! Rate $\&$ comment on articles 


\title{
Transport processes in random arrays of cylinders. II. Viscous flow
}

\author{
A. S. Sangani and C. Yao \\ Department of Chemical Engineering and Materials Science, Syracuse University, Syracuse, New York \\ $13244-1190$
}

(Received 6 May 1987; accepted 31 May 1988)

The method described in Part I [Phys. Fluids 31, 2426 (1988)] is extended to treat the problem of determining the permeability of random arrays of infinitely long cylinders. The results for the transverse and longitudinal permeabilities averaged over several configurations of random arrays of cylinders are presented as a function of the area fraction of the cylinders. A detailed comparison is made with the estimates of the permeability obtained by various approximate and asymptotic theories to determine their range of validity.

\section{INTRODUCTION}

In Part $I,{ }^{1}$ heat conduction through random arrays of parallel circular cylinders suspended in a continuous matrix phase was studied. The effective thermal conductivity of the composite medium for several random configurations of the cylinders was calculated and it was observed that the variance in the effective conductivity among the different configurations of the random arrays having the same area fraction and the thermal conductivities of the individual components of the composite medium is relatively small, making it meaningful to represent the effective thermal conductivity of the random arrays of cylinders as a function of the area fraction and the thermal conductivities of the individual components alone. We now examine the Stokes flow through random arrays of cylinders and determine the longitudinal and transverse permeabilities for several configurations of the random arrays of cylinders. It is found, once again, that the variance in the permeability of the arrays having the same area fraction $\phi$ is small for longitudinal permeability up to $\phi=0.7$ and for transverse permeability up to $\phi=0.5$ and hence the results obtained via the exact analysis could be used to assess the range of usefulness or validity of various approximate theories and asymptotic expressions proposed in the literature to estimate the permeability. Unlike the problem of determining the thermal conductivity, the bounds on the permeability are, in general, difficult to estimate and therefore no comparison with the bounds is made.

\section{GOVERNING EQUATIONS AND THE METHOD OF SOLUTION}

Consider Stokes flow through an array of cylinders defined in Part $\mathrm{I}$. The centers of $N$ cylinders within a representative unit cell are assumed to be held fixed at $\mathbf{R}^{(n)}$, $n=1,2, \ldots, N$. The fluid is incompressible and Newtonian, and the Reynolds number of the flow is small compared to unity. The governing equations at steady state are

$$
\begin{aligned}
& \frac{\partial p}{\partial x_{i}}=\mu \nabla^{2} u_{i}, \\
& \frac{\partial u_{i}}{\partial x_{i}}=0,
\end{aligned}
$$

$$
\begin{aligned}
& u_{i}=0, \quad \text { on } \partial D^{(n)}, \quad n=1,2, \ldots, N, \\
& \left\langle u_{i}\right\rangle=U_{i},
\end{aligned}
$$

along with the periodicity condition for the velocity $u_{i}$. Here $\mu$ is the viscosity of the fluid, $\partial D^{(n)}$ is the surface of the $n$th cylinder, and $U_{i}$ is the mean or the "superficial" velocity of the fluid. We wish to determine the permeability $K_{i j}$ defined by the relation

$$
-\mu U_{i}=\left\langle a^{2}\right\rangle K_{i j} P_{j},
$$

where $P_{j}$ is the mean pressure gradient in the fluid and $\left\langle a^{2}\right\rangle$ is the mean-squared radius of the particles. The cylinders are aligned along the $x_{3}$ axis and hence $K_{33}$ is referred to as the longitudinal permeability while $K_{11}, K_{12}$, and $K_{22}$ are referred to as the components of the transverse permeability. The methods of solution for determining these quantities are described separately.

\section{A. Transverse flow}

In this case, the flow is in the $x_{1}-x_{2}$ plane, i.e., perpendicular to the axes of the cylinders. To determine the velocity field, it is convenient to solve first the governing equations in terms of a streamfunction $\psi$ and vorticity $\omega$ in the fluid satisfying

$$
\nabla^{2} \psi=\omega, \quad \nabla^{2} \omega=0 .
$$

Since the vorticity satisfies the Laplace equation, a general solution for $\omega$ can be obtained in terms of various derivatives of $S_{1}$ as in Part I. Thus, to within an arbitrary constant,

$$
\omega(\mathbf{r})=\sum_{s=1}^{N} \sum_{n=1}^{\infty} \sum_{m=0}^{1} A_{n m}^{(s)} \frac{\partial^{n}}{\partial x_{1}^{n-m} \partial x_{2}^{m}} S_{1}\left(\mathbf{r}-\mathbf{R}^{(s)}\right),
$$

and $\psi$ can be expressed as a particular solution of (5) plus the solution of the homogeneous equation, i.e., the Laplace equation. The particular solution of (5) can be obtained in terms of a function $S_{2}$ satisfying ${ }^{2}$

$$
\nabla^{2} S_{2}=S_{1},
$$

whereas the homogeneous solution can be expressed, once again, in terms of the derivatives of $S_{1}$. Thus a general expression for the streamfunction is 


$$
\begin{aligned}
\psi= & U_{1} x_{2}-U_{2} x_{1}+\sum_{s=1}^{N}\left[B_{00}^{(s)} S_{1}\right. \\
& +\sum_{n=1}^{\infty} \sum_{m=0}^{1}\left(A_{n m}^{(s)} \frac{\partial^{n} S_{2}}{\partial x_{1}^{n-m} \partial x_{2}^{m}}\right. \\
& \left.\left.+B_{n m}^{(s)} \frac{\partial^{n} S_{1}}{\partial x_{1}^{n-m} \partial x_{2}^{m}}\right)\right]
\end{aligned}
$$

where $U_{1}$ and $U_{2}$ are the components of the average velocity of the fluid. In (7), $S_{1}$ and $S_{2}$ must be evaluated at $\mathbf{r}-\mathbf{R}^{(s)}$. It may be noted that the above expression applies to the problems in which the mean fluid velocity is constant. For the situations in which the mean fluid velocity has a nonzero gradient, the first two terms in (7) must be replaced by the appropriate terms.

To complete the solution, the constants $A$ and $B$ appearing in (7) must be evaluated by applying the condition ( $2 \mathrm{a}$ ) to each of the particles. Differentiating (7) with respect to $x_{1}$ and $x_{2}$, and substituting the resulting expressions for $u_{1}$ and $u_{2}$ into (1), we obtain

$$
\begin{aligned}
u_{1}= & U_{1}+\sum_{s=1}^{N}\left[B_{00}^{(s)} \frac{\partial S_{1}}{\partial x_{2}}\right. \\
& +\sum_{n=1}^{\infty} \sum_{m=0}^{1}\left(A_{n m}^{(s)} \frac{\partial^{n+1} S_{2}}{\partial x_{1}^{n-m} \partial x_{2}^{m+1}}\right. \\
& +B_{n m}^{(s)} \frac{\partial^{n+1} S_{1}}{\left.\left.\partial x_{1}^{n-m} \partial x_{1}^{m+1}\right)\right]} \\
u_{2}= & U_{2}-\sum_{s=1}^{N}\left[B_{\infty}^{(s)} \frac{\partial S_{1}}{\partial x_{1}}\right. \\
& +\sum_{n=1}^{\infty} \sum_{m=0}^{1}\left(A_{n m}^{(s)} \frac{\partial^{n+1} S_{2}}{\partial x_{1}^{n-m+1} \partial x_{2}^{m}}\right. \\
& \left.\left.+B_{n m}^{(s)} \frac{\partial^{n+1} S_{1}}{\partial x_{1}^{n-m+1} \partial x_{2}^{m}}\right)\right], \\
\frac{1}{\mu} p= & \sum_{s=1}^{N}\left[A_{11}^{(s)}\left(\frac{4 \pi x_{1}}{h^{2}}-\frac{\partial S_{1}}{\partial x_{1}}\right)+A_{10}^{(s)}\left(-\frac{4 \pi x_{2}}{h^{2}}+\frac{\partial S_{1}}{\partial x_{2}}\right)\right. \\
& +\sum_{n=2}^{\infty} \sum_{m=0}^{1} A_{n m}^{(s)} \frac{\partial^{n} S_{1}}{\left.\partial x_{1}^{n-m-1} \partial x_{2}^{m+1}\right]}
\end{aligned}
$$

where use has been made of

$$
\nabla^{2} S_{1}(r)=4 \pi\left(\frac{1}{h^{2}}-\sum_{r_{L}} \delta\left(r-\mathbf{r}_{\mathbf{L}}\right)\right) .
$$

Here $h$ is the size of the unit cell and $r_{L}$ are the lattice vectors as defined in Part I. The no-slip boundary condition (2a) may be expressed equivalently as

$$
\int_{0}^{2 \pi} \mathbf{u}(\mathbf{r}) e^{i l \theta} d \theta=0, \quad \text { on } \partial D^{(n)}, \quad l=1,2, \ldots,
$$

where $i=\sqrt{-1}$ and $\theta$ is the angle between $\mathbf{r}-\mathbf{R}^{(n)}$ and the $x_{1}$ axis. To evaluate the integrals in (12), we decompose $\mathbf{u}$ given by (8) and (9) into two parts,

$$
\mathbf{u}=\mathbf{u}_{\mathrm{s}}+\mathbf{u}_{\mathrm{r}},
$$

where $u_{s}$ is singular at $r=R^{(n)}$ and $u_{r}$ is the remainder which is nonsingular at $\mathbf{R}^{(n)}$. Thus, for example, $\mathbf{u}_{\mathbf{s}}$ consists of the terms containing $A^{(s)}$ and $B^{(s)}$ in (8) and (9) with $s=n$. The contribution to the integral in (12) from the non- singular part can be evaluated by making use of the following theorem.

Theorem: Let $u_{r}$ be a function satisfying $\nabla^{4} u_{r}=0$ and be nonsingular for $\mathbf{r}$ inside a circle centered at $\mathbf{r}^{(n)}$. Then,

$$
\begin{aligned}
& \frac{1}{2 \pi} \int_{0}^{2 \pi} \mathbf{u}_{\mathrm{r}}(\mathbf{r}) \cos l \theta d \theta \\
& =\frac{1}{2}\left(1+\delta_{l 0}\right) \frac{a^{l}}{l !}\left[\left(\frac{\partial}{\partial x_{1}}\right)^{l}-\frac{l}{4}\left(\frac{\partial}{\partial x_{1}}\right)^{l-2} \nabla^{2}\right. \\
& \left.\quad+\frac{a^{2}}{4(1+l)}\left(\frac{\partial}{\partial x_{1}}\right)^{l} \nabla^{2}\right] \mathbf{u}_{\mathrm{r}}\left(\mathbf{r}^{(n)}\right), \\
& \frac{1}{2 \pi} \int_{0}^{2 \pi} \mathbf{u}_{\mathrm{r}}(\mathbf{r}) \sin l \theta d \theta \\
& \left.=\frac{1}{2} \frac{a^{l}\left[\left(\frac{\partial}{l !}\right)^{l-1}\left(\frac{\partial}{\partial x_{1}}\right)-\frac{l-2}{4}\left(\frac{\partial}{\partial x_{2}}\right)^{l-3}\left(\frac{\partial}{\partial x_{2}}\right) \nabla^{2}\right.}{}+\frac{a^{2}}{4(1+l)}\left(\frac{\partial}{\partial x_{1}}\right)^{l-1}\left(\frac{\partial}{\partial x_{2}}\right) \nabla^{2}\right] \mathbf{u}_{\mathrm{r}}\left(\mathbf{r}^{(n)}\right),
\end{aligned}
$$

where $a$ is the radius of the circle. In (14) and (15), the terms with a negative order of differentiation with respect to $x_{1}$ must be set equal to zero. The integration is assumed to be along the circumference, i.e., for $\left|\mathbf{r}-\mathbf{R}^{(n)}\right|=a$. The proof of (14) and (15) is given in Appendix A, where a similar result for a corresponding integral on the spherical surfaces is also given.

In order to evaluate the various derivatives of $\mathbf{u}_{\boldsymbol{r}}$ needed in making use of (14) and (15), and also to evaluate the contribution of the singular part $u_{s}$ to the integral in (12), we need the following expansions of $S_{1}$ and $S_{2}$ (see Ref. 3 ):

$$
\begin{aligned}
S_{1}\left(\mathbf{r}-\mathbf{r}^{(p)}\right)= & -2 \log s-\bar{c}+\frac{\pi s^{2}}{h^{2}}+\sum_{n=1}^{\infty} c_{2 n} s^{4 n} \cos 4 n \theta \\
S_{2}\left(\mathbf{r}-\mathbf{r}^{(p)}\right)= & \frac{s^{2}}{2}(1-\log s)+\bar{c}_{2}-\frac{\bar{c} s^{2}}{4}+\frac{\pi s^{4}}{16 h^{2}} \\
& +\sum_{n=1}^{\infty}\left(\tilde{c}_{2 n} s^{2}+b_{2 n}\right) s^{4 n} \cos 4 n \theta
\end{aligned}
$$

where $s=\left|\mathbf{r}-\mathbf{r}^{(p)}\right|, \theta$ is the angle between $\mathbf{r}-\mathbf{r}^{(n)}$ and the $x_{1}$ axis, and $\bar{c}, \bar{c}_{2}, c_{2 n}, \tilde{c}_{2 n}$, and $b_{2 n}$ are evaluated in Ref. 3 . It may be noted that, for $p=n$, the first term on the right-hand side of (16) is singular inside the $n$th cylinder and thus $u_{8}$ consists of the terms arising from the differentiation of this singular term. Similarly the first term on the right-hand side of (17) also gives singular terms upon differentiation. For $p \neq n$, all the terms on the right-hand sides of (16) and (17) are nonsingular and the formulas given in Appendix B of this paper and the appendix of Part I may be used to evaluate the derivatives of $S_{1}$ and $S_{2}$ at any point in the unit cell provided that the series obtained by the differentiation of (16) and (17) are termwise convergent, i.e., for a sufficiently small $s$ or for the order of differentiation not greater than, say, 4. For the higher-order derivatives or for larger $s$, the alternate expressions given in the above appendices may be used to evaluate the integrals in (12) to a high accuracy.

Upon application of (12) for various $l$ to each of the $N$ particles in the unit cell, an infinite set of linear equations in $A$ and $B$ results, which may be truncated to a finite set by retaining only those equations with $l \leqslant L$ in (12) and the un- 
knowns $A_{n m}$ with $n \leqslant L+1$ and $B_{n m}$ with $n \leqslant L$. This results in a total of $(4 L+2) N$ linear equations in a total of $(4 L+1) N$ unknowns. It can be easily shown that, because of the equation of continuity ( $1 b$ ), the above equations are not linearly independent and that one of the four equations for $l=1$ [cf. (12)] may be discarded from the above set of equations resulting in a total of $(4 L+1) N$ equations in an equal number of unknowns. On solving these equations and on making use of the relations given below [cf. (26)], the transverse permeability of the array with specified $\mathbf{R}^{(n)}$ can be determined. Now $L$ may be systematically increased until no significant change in the calculated values of the permeability is observed. The stress tensor $\sigma_{i j}$ defined by

$$
\sigma_{i j}=-p \delta_{i j}+\mu\left(\frac{\partial u_{i}}{\partial x_{j}}+\frac{\partial u_{j}}{\partial x_{i}}\right)
$$

can be computed from (8)-(10) and, on making use of (11), it can be shown that

$$
\begin{aligned}
\frac{\partial \sigma_{1 j}}{\partial x_{j}}= & -4 \pi \mu \sum_{s=1}^{N}\left[\left(A_{11}^{(s)}+B_{\infty 0}^{(s)} \frac{\partial}{\partial x_{2}}\right.\right. \\
& \left.\left.+\sum_{n=1}^{\infty} \sum_{m=0}^{1} B_{n m}^{(s)} \frac{\partial^{n+1}}{\partial x^{n-m} \partial x_{2}^{m+1}}\right) \delta\left(\mathbf{r}-\mathbf{r}^{(s)}\right)\right] \\
\frac{\partial \sigma_{2 j}}{\partial x_{j}}= & 4 \pi \mu \sum_{s=1}^{N}\left[\left(A_{10}^{(s)}+B_{\infty 0}^{(s)} \frac{\partial}{\partial x_{1}}\right.\right. \\
& +\sum_{n=1}^{\infty} \sum_{m=0}^{1} B_{n m}^{(s)} \frac{\partial^{n+1}}{\partial x_{1}^{n-m+1} \partial x_{2}^{m}} \\
& \left.\left.+\sum_{n=2}^{\infty} \sum_{m=0}^{1} A_{n m}^{(s)} \frac{\partial^{n-1}}{\partial x_{1}^{n-m-1} \partial x_{2}^{m}}\right) \delta\left(\mathbf{r}-\mathbf{r}^{(s)}\right)\right]
\end{aligned}
$$

The force on each particle can be easily calculated from (19) and $(20)$ by using

$$
F_{i}^{(s)}=\int_{\partial D^{(s)}} \frac{\partial \sigma_{i j}}{\partial x_{j}} d V,
$$

which yields

$$
\begin{aligned}
& F_{1}^{(s)}=-4 \pi \mu A_{11}^{(s)}, \\
& F_{2}^{(s)}=4 \pi \mu A_{10}^{(s)} .
\end{aligned}
$$

Similarly the torque exerted by the fluid on the sth cylinder is related to $B_{00}$ and $A_{20}$ by

$$
L_{3}^{(s)}=\epsilon_{3 j k} \int_{\partial D^{(s)}} \frac{\partial \sigma_{j l}}{\partial x_{l}} x_{k}^{\prime} d V=4 \pi \mu\left(2 B_{00}^{(s)}+A_{20}^{(s)}\right),
$$

where $x_{k}^{\prime}=x_{k}-R_{k}^{(s)}$. Finally, the mean pressure gradient in the fluid is given by

$$
P_{i} h^{2}=\sum_{n=1}^{N} F_{i}^{(n)}
$$

which on combining with (3), (22), and (23) gives the following expressions for the components of the transverse permeability:

$$
K_{11} \sum_{s=1}^{N} A_{11}^{(s)}-K_{12} \sum_{s=1}^{N} A_{10}^{(s)}=\frac{N U_{1}}{4 \phi},
$$

$$
K_{21} \sum_{s=1}^{N} A_{11}^{(s)}-K_{22} \sum_{s=1}^{N} A_{10}^{(s)}=\frac{N U_{2}}{4 \phi} .
$$

The determination of the components of the transverse permeability for a given configuration of $N$ cylinders, which is in general anisotropic, requires solving two problems. In the first, $U_{1}=1$ and $U_{2}=0$, and in the second, $U_{1}=0$ and $U_{2}=1$. Of course $K_{12}=K_{21}$ is automatically satisfied and hence only three independent equations result from (26) upon solving the above two problems. As in Part I, the results for the permeability are given in terms of $K_{m}, K_{d}$, and $|\psi|$, which are related to the components of the transverse permeability tensor along the major and minor axes. It may be noted that the torque on the individual particle or on the entire array is, in general, nonzero and may be evaluated, if necessary, by making use of (24).

\section{B. Longitudinal flow}

When the flow is along the $x_{3}$ axis, i.e., along the axes of the cylinders, the only nonzero component of the velocity is $u_{3}$, and the equations of motion reduce to the Poisson equation:

$$
\nabla^{2} u_{3}=\frac{1}{\mu} \frac{d p}{d x_{3}}
$$

where $d p / d x_{3}$ is a constant. It may be noted that for a steady laminar longitudinal flow, the inertial terms in the equations of motion are identically zero and hence (27) is valid even for nonzero Reynolds numbers. Once again, since $S_{1}$ is the singular solution of the Poisson equation, the solution of (27) can be obtained in terms of the derivatives of $S_{1}$. We write

$$
\begin{aligned}
\boldsymbol{u}_{3}(\mathbf{r})= & U_{0}+\sum_{s=1}^{N}\left(C_{\infty 0}^{(s)}+\sum_{n=1}^{\infty} \sum_{m=0}^{1} C_{n m}^{(s)}\right. \\
& \left.\times \frac{\partial^{n}}{\partial x_{1}^{n-m} \partial x_{2}^{m}}\right) S_{1}\left(\mathbf{r}-\mathbf{r}^{(s)}\right),
\end{aligned}
$$

and, in view of (27) and (11),

$$
\frac{1}{\mu} \frac{d p}{d x_{3}}=\frac{1}{\mu} P_{3}=\frac{4 \pi}{h^{2}} \sum_{s=1}^{N} C_{00}^{(s)} \text {. }
$$

Here $U_{0}$, as shown in Appendix $\mathrm{C}$, is related to the mean velocity $U_{3}$ by

$$
U_{0}=U_{3}+\phi\left(1-\frac{\phi}{2}\right) \frac{1}{N} \sum_{s=1}^{N} C_{\infty 0}^{(s)}
$$

The constants $C$ are evaluated from the no-slip boundary conditions in the same manner as described in Sec. II A. The longitudinal permeability $K_{33}$ can be calculated from

$$
K_{33}=\frac{-U_{3} N}{4 \phi \Sigma_{s=1}^{N} C_{00}^{(s)}} \text {. }
$$

\section{RESULTS}

As in Part I, the method of solution and the numerical scheme for calculating the transverse and longitudinal permeabilities were first checked by calculating the permeabilities of the periodic arrays of cylinders by choosing $\mathbf{R}^{(s)}$ to coincide with a lattice whose dimensions are an intergral fraction of $h$. The results were almost indistinguishable from 
those of Sangani and Acrivos ${ }^{4}$ and Sparrow and Loeffler ${ }^{5}$ for the complete range of area fractions of the cylinders with $n=1,4$, and 9 . The convergence of the numerical results for the longitudinal permeability was very rapid whereas terms up to $L=15$ (61 unknowns per particle) were needed to converge the results for the transverse permeability for $\phi=0.7$. The calculations of the permeability were then made on the random configurations of the cylinders generated in Part $I$.

Table I shows the convergence of the longitudinal permeability $K_{33}$ for the random arrays with $N=16$. The total number of unknowns in this case is $(2 L+1) N$. The convergence is very rapid, and the subsequent calculations were made with $L=14$ for $\phi>0.5$. The results of averaging over several (8-10) configurations are shown in Table II where the corresponding results for the longitudinal permeability of the square arrays are also given. It is interesting to note that the longitudinal permeability of the random arrays is greater than that of the square arrays for all $\phi$, the value at $\phi=0.1$ differing almost by a factor of 2 . The results with $N=9$ and $\phi=0.7$ are also given in Table II, and it may be noted that the results obtained with $N=9$ and $N=16$ agree with each other.

Table III shows the convergence of the numerical results for the components $K_{m}, K_{d}$, and $|\psi|$ of the transverse permeability tensor. The number of unknowns is $(4 L+1) N$. The convergence at higher area fractions is slow and this limited the calculations to $N=9$ for $\phi=0.5$ and 0.7 . A few calculations were carried out with $N=16$ but no significant change in the degree of anisotropy, as determined by the ratio $K_{d} / K_{m}$, was observed. The results of the averaging over several configurations (5-8) of the random arrays are shown in Table IV. The pressure drop in the fluid as it moves through a "gap" between two neighboring particles increases very rapidly as the "gap" between the particles becomes small and the mean pressure gradient appears to become very sensitive to the detailed configuration of the particles resulting in a large degree of anisotropy in each of the configurations at higher area fractions. Thus large computational efforts that would be required for detemining the transverse permeability at larger $\phi$ and $N$ do not appear worthwhile.

Although a significant degree of anisotropy is observed in the transverse permeability tensor, it is interesting to note that $K_{m}$ for the random arrays is not significantly different from that for the square arrays, at least for $\phi \leqslant 0.5$.

TABLE I. Convergence of the numerical results for the longitudinal permeability $K_{33}$ of the random arrays.

\begin{tabular}{ccl}
\hline$\phi$ & $L$ & \multicolumn{1}{c}{$K_{33}$} \\
\hline & & \\
0.1 & 2 & 4.725 \\
& 4 & 4.593 \\
0.7 & 6 & 4.575 \\
& 6 & $1.727 \times 10^{-2}$ \\
& 10 & $1.492 \times 10^{-2}$ \\
& 20 & $1.500 \times 10^{-2}$ \\
\hline
\end{tabular}

TABLE II. The longitudinal permeability $K_{33}$ of the random (RA) and square (SA) arrays of cylinders.

\begin{tabular}{|c|c|c|c|}
\hline$\phi$ & $N$ & $\begin{array}{c}K_{33} \\
(\text { RA }) \\
\text { mean } \pm \text { s.d. }\end{array}$ & $\begin{array}{c}K_{33} \\
(\mathbf{S A})\end{array}$ \\
\hline 0.1 & 16 & $4.94 \pm 0.7$ & 2.55 \\
\hline 0.3 & 16 & $0.45 \pm 0.04$ & 0.235 \\
\hline 0.5 & 16 & $(6.88 \pm 1.1) 10^{-2}$ & $4.45 \times 10^{-2}$ \\
\hline 0.7 & 16 & $(1.4 \pm 0.2) 10^{-2}$ & $0.94 \times 10^{-2}$ \\
\hline 0.7 & 9 & $(1.25 \pm 0.2) 10^{-2}$ & $0.94 \times 10^{-2}$ \\
\hline
\end{tabular}

\section{COMPARISON WITH OTHER METHODS}

The results for the longitudinal and transverse permeabilities presented in Sec. III are compared in this section with the asymptotic expressions and the approximate theories.

\section{A. Asymptotic expressions}

\section{The low area fraction limit}

Howells ${ }^{6}$ has analyzed theoretically the permeability of the random arrays of cylinders by taking into account the pairwise interaction between cylinders. His analysis assumed the radial distribution function to be unity for distances greater than one particle diameter, and zero otherwise. The results for the permeability to $O(\phi)$ were given in terms of two self-consistent equations [cf. (7.10) and (7.13) in Ref. 6], and the results of solving these two equations were presented graphically (cf. Fig. 7, Ref. 6) in terms of $M_{l}$ and $M_{t}$, which are related to the longitudinal and transverse permeabilities by

$$
\begin{aligned}
& K_{33}=M_{l}(1-\phi) / 2 \phi, \\
& K_{m}=M_{t} / 4 \phi .
\end{aligned}
$$

Table $\mathrm{V}$ shows the comparison of the numerical results of Sec. III with the estimates given by Howells. We see that, although the longitudinal permeability calculated with $N=16$ and averaged over ten configurations differs from the Howells estimate by approximately $15 \%$ at $\phi=0.1$, it is within approximately one standard deviation from the mean, and the comparison between the two methods must be therefore regarded as good. Similarly the agreement between the two methods in the case of the transverse permeability must also be regarded as good for $\phi \leqslant 0.5$.

TABLE III. Convergence of the numerical results for the transverse permeability of the random arrays of cylinders.

\begin{tabular}{ccllll}
\hline \hline$\phi$ & $L$ & \multicolumn{1}{c}{$K_{m}$} & \multicolumn{1}{c}{$K_{d}$} & $|\psi|$ & $N$ \\
\hline \multirow{4}{*}{0.1} & 2 & 1.684 & 0.179 & 15.4 & \\
& 4 & 1.690 & 0.179 & 15.0 & 16 \\
& 7 & 1.691 & 0.179 & 15.1 & \\
0.5 & 10 & $0.95 \times 10^{-2}$ & $0.42 \times 10^{-2}$ & 43 & \\
& 15 & $1.12 \times 10^{-2}$ & $0.37 \times 10^{-2}$ & 42 & 9 \\
& 18 & $1.10 \times 10^{-2}$ & $0.37 \times 10^{-2}$ & 41 & \\
$0.7^{a}$ & 10 & $0.11 \times 10^{-4}$ & $\ldots$ & $\cdots$ & 4 \\
& 15 & $0.33 \times 10^{-3}$ & $\ldots$ & $\cdots$ & 4 \\
& 18 & $0.33 \times 10^{-3}$ & $\ldots$ & $\ldots$ & \\
\hline \hline
\end{tabular}

"For a square array. 
TABLE IV. Results for the transverse permeability.



${ }^{3}$ Corresponds to a single configuration of the array.

\section{The high area fraction IImit}

As the area fraction of the cylinders approaches its maximum value, the longitudinal permeability approaches a constant value, and an asymptotic analysis for this case is not feasible. The same is also true for the case of the transverse permeability if there exists a "channeling" at the high area fractions, i.e., if the random configurations of the cylinders at the maximum areal fraction permit open paths through which the fluid can flow from one end of the porous medium to the other without much resistance. The case of a special class of random arrays described in Sec. IV C in Part I in which there is no "channeling" and the "gaps" between the neighboring particles decrease uniformly as $\phi \rightarrow \phi_{t}$ is amenable to an asymptotic analysis. It can be shown using a lubrication-type analysis that for such arrays, the transverse permeability is given by

$$
K_{m}=(8 \sqrt{2} / 9 Z)\left[1-\left(\phi / \phi_{t}\right)^{1 / 2}\right]^{5 / 2}, \quad \phi \rightarrow \phi_{t},
$$

where $Z$ is the average coordination number.

It is interesting to compare how the $K_{m}$ estimated for such random arrays compare with the $K_{m}$ of the random arrays used in our study. As seen in Table V, (34) with $\phi=0.82$ and $Z=4.2$ (cf. the discussion in Part I, Sec. IV C) provides an excellent estimate of $K_{m}$ for the random arrays simulated in the present study.

It may be noted that (34) also provides an excellent estimate of the transverse permeabilities of the square and hexagonal arrays for $\phi \geqslant 0.2{ }^{4}$ The values of $Z$ and $\phi_{t}$ for these two arrays are, respectively, 4 and $\pi / 4=0.79$ for the square and 6 and $\pi / 2 \sqrt{ } 3=0.907$ for the hexagonal arrays.

\section{B. Approximate methods}

As in Part I, these are methods in which, rather than solving the detailed flow field around many particles distributed throughout the space, one solves for the fluid flow around a single particle immersed in a medium with suitably defined properties.

\section{Transverse permeability}

We first discuss two approximate methods for estimating the transverse permeability in which the only information needed is the area fraction of the cylinders in the array. Both of these methods can be described by the following equations:

$$
\begin{aligned}
& \boldsymbol{\nabla} \cdot \mathbf{u}=0, \\
& \mu \nabla^{2} \mathbf{u}-\boldsymbol{\nabla} p=\rho(r) B \mathbf{u}, \\
& \mathbf{u} \rightarrow \mathbf{U}, \quad r \rightarrow \infty, \\
& \mathbf{u}=0, \quad r=1,
\end{aligned}
$$

where the radius of the cylinder is assumed, without loss of generality, to be unity. In Brinkman's approximation $\rho(r)=\phi$ for all $r$ whereas in the self-consistent scheme

$$
\rho(r)= \begin{cases}0, & 1 \leqslant r \leqslant \phi^{-1 / 2} \\ \phi, & r>\phi^{-1 / 2}\end{cases}
$$

The constant $B$ in the above equation is related to the transverse permeability to be estimated and it is determined in a self-consistent manner so as to satisfy

$$
\begin{aligned}
& K_{m}=\mu / \phi B, \\
& \mathrm{~F}=B \mathrm{U},
\end{aligned}
$$

where $\mathbf{F}$ is the force on the particle as calculated by solving (35) and (36) for an assumed value of $B$. The Brinkman estimate is given by ${ }^{6}$

$$
2 \phi\left[2 K_{1}(\alpha) / \alpha K_{0}(\alpha)+1\right]=1, \quad \alpha=K_{m}^{-1 / 2},
$$

\begin{tabular}{|c|c|c|c|c|c|}
\hline \multirow[b]{3}{*}{$\phi$} & \multicolumn{2}{|c|}{$K_{33}$} & \multicolumn{2}{|c|}{$\overline{K_{m}}$} & \multirow[b]{3}{*}{ (34) } \\
\hline & exact & & exact & & \\
\hline & mean $\pm s . d$. & Howells $^{\mathrm{a}}$ & mean $\pm s . d$ & Howells & \\
\hline 0.1 & $4.94 \pm 0.7$ & 5.8 & $1.67 \pm 0.12$ & 1.79 & 1.03 \\
\hline 0.3 & $0.45+0.04$ & 0.64 & $(10.2 \pm 0.8) 10^{-2}$ & 0.108 & $9.8 \times 10^{-2}$ \\
\hline 0.5 & $(6.88 \pm 1.1) 10^{-2}$ & 0.13 & $(9.4 \pm 1.7) 10^{-3}$ & $7.7 \times 10^{-3}$ & $13 \times 10^{-3}$ \\
\hline 0.7 & $(1.4 \pm 0.2) 10^{-2}$ & $1.5 \times 10^{-3}$ & $(0.8-1.2) 10^{-3}$ & $\ldots$ & $0.7 \times 10^{-3}$ \\
\hline
\end{tabular}

whereas the self-consistent estimate is given by

$\frac{2 K_{1}(R)}{K_{0}(R)}+\frac{R}{2} \frac{R^{2} K_{1}(R)}{4 K_{0}(R)} \log \phi-\frac{R^{3}}{8}(\phi-\log \phi-1)=0$,

where $R=\left(\phi K_{m}\right)^{-1 / 2}$ and $K_{0}$ and $K_{1}$ are the modified Bessel functions. Both of these estimates are $O(\log \phi / \phi)$ as $\phi \rightarrow 0$ and hence agree with Howells ${ }^{6}$ analysis for the low area fractions of the cylinders. The $O(1)$ constants, however, are dif-

TABLE V. Comparison of the rigorous calculations for the longitudinal and transverse permeability with the asymptotic formulas.

"See Ref. 6. 
TABLE VI. Comparison of the rigorous calculations for the transverse permeability with various approximate theories."

\begin{tabular}{|c|c|c|c|c|c|c|}
\hline$\phi$ & Array & Exact & $\mathrm{Br}$ & $\mathrm{SC}$ & MB & MA \\
\hline \multirow[b]{2}{*}{0.1} & RA & $1.67 \pm 0.12$ & 1.3 & 3.0 & 1.63 & 1.57 \\
\hline & SA & 1.27 & 1.3 & 3.0 & 1.99 & 1.40 \\
\hline \multirow[t]{2}{*}{0.3} & SA & $10.2 \times 10^{-2}$ & $9.7 \times 10^{-2}$ & 0.47 & 0.205 & 0.120 \\
\hline & RA & $(9.47 \pm 1.7) 10^{-3}$ & 0 & 0.14 & $12 \times 10^{-3}$ & $11.2 \times 10^{-3}$ \\
\hline 0.5 & SA & $11.8 \times 10^{-3}$ & 0 & 0.14 & $23 \times 10^{-3}$ & $13.2 \times 10^{-3}$ \\
\hline 0.7 & HA & $16.2 \times 10^{-4}$ & & $3.8 \times 10^{-2}$ & $\cdots$ & $\cdots$ \\
\hline
\end{tabular}

${ }^{\mathrm{a}} \mathrm{RA}=$ random array, $\mathrm{SA}=$ square array, $\mathrm{Br}=$ Brinkman's approximation, $\mathrm{SC}=$ self-consistent scheme, $\mathrm{MB}=$ method $\mathrm{B}$, and $\mathrm{MA}=$ method $\mathrm{A}$.

ferent and hence the estimates obtained from the above two methods differ significantly at $\phi=0.1$ as seen in Table VI where a comparison of the estimates obtained by these two methods is made with the exact results for the square and random arrays of cylinders. The Brinkman estimates appear to be reasonably good for the lower area fractions of the cylinders in random arrays but overestimates the permeability of the square arrays. At $\phi \geqslant 0.5$, the Brinkman model gives unrealistic negative permeabilities. The self-consistent scheme provides reasonable estimates of the transverse permeabilities for $\phi \geqslant 0.3$.

The above two methods do not employ any additional information on the geometry of the array other than the area fraction of the cylinders. In recent years, approximate methods have been proposed which, in addition to $\phi$, require knowledge of the probability function $\rho(r)$ of the array. It is of interest to determine how successfully these methods can use knowledge of $\rho(r)$, if available, in giving better estimates of the permeability. We shall discuss here two such methods which are somewhat equivalent to methods $\mathrm{A}$ and $\mathrm{B}$ described in Part $\mathbf{I}$.

According to the method proposed by Buyevich and Shchelchkova ${ }^{7}$ (referred to as method B), both the permeability and the viscosity of the medium are functions of the radial positions and the disturbance flow satisfies, in addition to (35a),

$$
\begin{aligned}
& -\frac{\partial \hat{p}}{\partial x_{i}}+\frac{\partial}{\partial x_{j}}\left[\mu_{a}\left(\frac{\partial \hat{u}_{i}}{\partial x_{j}}+\frac{\partial \hat{u}_{j}}{\partial x_{i}}\right)\right]-B \rho(r) \hat{u}_{i}=0, \\
& u_{i}=\hat{u}_{i}+u_{i}^{\infty}, \quad p=\hat{p}+p^{\infty}, \\
& \hat{u}_{i}=-u_{i}^{\infty}, \quad r=1, \\
& \hat{u}_{i}=0, \quad r \rightarrow \infty \\
& \mu_{a}=\mu+\rho(r)\left(A-B^{*}\right),
\end{aligned}
$$

where $\rho(r)$ is the probability density function for the solid phase as defined in Part $I$. The constants $A, B$, and $B^{*}$ are to be determined in a self-consistent manner from the following relations:

$$
\begin{aligned}
& F_{i}=\pi\left[B u_{i}^{\infty}(0)+B^{*} \nabla^{2} u_{i}^{\infty}(0)\right], \\
& S_{i j}=\pi\left[B_{1} e_{i j}^{\infty}(0)+B_{2} \nabla^{2} e_{i j}^{\infty}(0)\right], \quad e_{i j} \equiv \frac{\partial u_{i}}{\partial x_{j}}+\frac{\partial u_{j}}{\partial x_{i}} \\
& A=B_{1}+\frac{\phi B}{\mu_{a}^{\infty}} B_{2}
\end{aligned}
$$

where $F_{i}$ and $S_{i j}$ are the force and stresslet on the particle as calculated by solving (42)-(44) for the assumed values of $A$, $B^{*}$, and $B$, i.e.,

$$
\begin{aligned}
& F_{i}=\int \sigma_{i j} n_{j} d A, \\
& S_{i j}=\frac{1}{2} \int\left(x_{i} \sigma_{j k}+x_{j} \sigma_{i k}\right) n_{k} d A,
\end{aligned}
$$

where $\sigma_{i j}$ is the stress tensor, the integral is along the surface of the particle, and $n_{k}$ is the unit outward normal on the surface. In general, the determination of the constants $A, B$, $B^{*}$, and thus $K_{m}$ from (38), requires iteratively solving four problems simultaneously in which (i) $u_{i}^{\infty} \neq 0$; (ii) $\nabla^{2} u_{i}^{\infty}(0)$ $\neq 0$; (iii) $e_{i j}^{\infty}(0) \neq 0$; and (iv) $\nabla^{2} e_{i j}^{\infty}(0) \neq 0$. Fortunately, it is possible to derive a reciprocal theorem

$$
\int \hat{u}_{i} \hat{\sigma}_{i j}^{\prime} n_{j} d A=\int \hat{u}_{i}^{\prime} \hat{\sigma}_{i j} n_{j} d A
$$

where both $\hat{u}_{i}$ and $\hat{u}_{i}^{\prime}$ satisfy (35a) and (42). The use of (51) allows us to determine $A, B$, and $B^{*}$ by solving only two flow problems: (i) the uniform flow, $u_{i}^{\infty}=\delta_{i 1}$; and (ii) the shear flow, $e_{i j}^{\infty}=\delta_{i 1} \delta_{j 2}+\delta_{i 2} \delta_{j 1}$. The self-consistent calculations for the case of constant permeability of the medium have been made by several authors. For example, Howells ${ }^{6}$ and Speilman and Goren ${ }^{8}$ have considered the case of cylindrical particles and Howells ${ }^{6}$ and Kim and Russe ${ }^{9}$ have considered the case of spherical particles. In such cases, it is possible to derive an analytical expression for the drag on the sphere. However, it is not possible to derive an expression for the force when the permeability of the medium is not uniform and the numerical scheme presented in Appendix D must then be used to obtain the estimates of the force.

The estimates of the transverse permeability obtained by this scheme are shown in Table VI, where we see that the method provides better estimates of $K_{m}$ for the random arrays as compared to Brinkman's or the self-consistent methods. However, the method fails to utilize the information on $\rho(r)$ effectively in that, for instance, at $\phi=0.1$ the values of $K_{m}$ predicted by this method are higher for the square arrays compared to those for the random arrays, whereas the rigorous calculations of Sec. III suggest that the opposite is the case, i.e., $K_{m}$ for the square arrays are lower than those for the random arrays. The equations to be solved in estimating $K_{m}$ by method B become stiff for $\phi>0.3$ and, consequently, reliable estimates of $K_{m}$ by this method are difficult to obtain 
for larger values of $\phi$. Of course, an asymptotic analysis of these equations could then be made to estimate $K_{m}$ at large $\phi$ by this method but, in view of the failure of the method to use properly the information on $\rho(r)$ as mentioned above, such an effort is unwarranted.

In method A, instead of solving for the distribution flow field $\hat{u}_{i}$ and $\hat{p}$, equations similar to (42)-(48) are solved for the "actual" flow field $u_{i}$ and $p$. Unfortunately, in this case it is not possible to derive a simple reciprocal theorem similar to (51) for the resulting governing equations. Besides, in view of the relatively complex scheme of computations that becomes necessary in trying to estimate both the permeability and the apparent viscosity of the porous medium simultaneously in a self-consistent manner, it is desirable to try a simpler approximate method. Thus, in lieu of (42), the following equation was solved:

$$
-\frac{\partial p}{\partial x_{i}}+\frac{\partial}{\partial x_{j}}\left[\mu_{a}\left(\frac{\partial u_{i}}{\partial x_{j}}+\frac{\partial u_{j}}{\partial x_{i}}\right)\right]-B \rho(r) u_{i}=0,
$$

with the boundary conditions

$$
\begin{aligned}
& u_{i}=U_{i} \quad \text { (a constant), } \quad r \rightarrow \infty, \\
& u_{i}=0, \quad r=1,
\end{aligned}
$$

and $A$ and $B *$ in (45) are assumed to be zero. In other words, the apparent viscosity $\mu_{a}$ of the medium was taken to be the same as that of the fluid. The results obtained by this method (referred to as method $A$ ) are shown in Table VI where they appear to give the estimates of the permeability that are better than those obtained from method B. The method also appears to utilize the information on $\rho(r)$ that is consistent with the rigorous calculations for the random and square arrays. Moreover, the computational scheme for estimating the permeability by this method is much simpler compared to that for method B. It is interesting to examine whether estimating the apparent viscosity by an independent method, say, by first solving the shear flow problem with $B$ in (52) taken to be zero, will provide better estimates of the permeability. Therefore, (52) was solved for several assumed nonzero values of $A-B^{*}$, i.e., $\mu_{a} \neq \mu$ and the permeability was estimated for the various assumed values of the apparent viscosity of the fluid. It was found that a better agreement with the exact calculations can be found only if the apparent viscosity of the porous medium is substantially less than the viscosity of the fluid, i.e., if $A-B^{*}<0$. The estimates of $A-B^{*}$ by method B were positive for all the cases examined and it is likely that other similar schemes would also estimate $\mu_{a}>\mu$. Thus it appears that estimating $\mu_{a}$ independently will very likely yield poor estimates of the permeability.

\section{Longitudinal permeability}

In this case the only nonzero component of the velocity is $u_{3}$ and the disturbance $\hat{u}_{3}$ in method $B$ satisfies

$$
-\frac{\partial \hat{p}}{\partial x_{3}}+\frac{\partial}{\partial x_{j}}\left(\mu_{a} \frac{\partial \hat{u}_{3}}{\partial x_{j}}\right)-\rho(r) B \hat{u}_{3}=0,
$$

with

$$
\hat{u}_{3}=0, \quad \text { as } r \rightarrow \infty \text {, }
$$

$$
\hat{u}_{3}=-u_{3}^{\infty}, \quad \text { at } r=1 .
$$

The force $F_{3}$ on the cylinder is related to $B$ and $B^{*}$ by

$$
F_{3}=\pi\left[B u_{3}^{\infty}(0)+B^{*} \nabla^{2} u_{3}(0)\right]
$$

and since the stresslet on the cylinder is identically zero,

$$
A=0 \text { and } \mu_{a}=\mu-\rho(r) B * \text {. }
$$

Furthermore, by the use of a reciprocal theorem for (54), it can be shown that

$$
\begin{aligned}
& B^{*}=\left[I_{0}(\beta)-1\right] / \beta^{2}+2 \mu I_{1}(\beta) / \beta, \\
& \beta^{2}=\phi B / \mu_{a}^{\infty}=\phi B /\left(\mu-\phi B^{*}\right),
\end{aligned}
$$

and

$$
K_{33}=(1-\phi) \mu / \phi B,
$$

where $I_{0}$ and $I_{1}$ are the modified Bessel functions. In method A, (54) is solved for $u_{3}$ instead of $\hat{u}_{3}$ and thus the boundary conditions (55) are reversed, and $B^{*}$ is taken as zero, i.e., $\mu_{a}=\mu$. Similarly in the self-consistent scheme and Brinkman's approximation (54) is solved, once again, for $u_{3}$ with $\rho(r)$ defined appropriately, i.e., $\rho(r)=\phi$ for all $r$ in the case of the Brinkman's approximation, and $\rho(r)$ given by (37) in the case of the self-consistent scheme. The comparison of the estimates obtained via these approximate schemes with $K_{33}$ calculated in Sec. III is given in Table VII. The Brinkman and self-consistent scheme estimates are given by, respectively,

$$
\frac{1-\phi}{2 \phi}=\frac{K_{1}\left(\alpha^{\prime}\right)}{\alpha^{\prime} K_{0}\left(\alpha^{\prime}\right)}, \quad \alpha^{\prime}=\frac{1}{K_{33}}
$$

and

$$
K_{33}=(\phi-1-\log \phi) / 4 \phi .
$$

The Brinkman estimate agrees well with $K_{33}$ of the random arrays at $\phi=0.1$ but the agreement is poor for the square arrays and for the random arrays at $\phi \geqslant 0.3$. The selfconsistent estimate is in reasonable agreement with the exact results at larger $\phi$. Method $B$, once again, overestimates the permeability and does not provide the estimates of $K_{33}$ consistent with the changes in $\rho(r)$. Method A, which does not correct for the viscosity of the medium, i.e., $\mu_{a}=\mu$, provides reasonably good estimates at lower $\phi$. At higher $\phi, \mu_{a}$ much higher than $\mu$ will be needed to obtain the estimates of $K_{33}$ by this method to agree with the exact values of $K_{33}$. Thus none of the approximate methods appear to give satisfactory estimates of the longitudinal permeability for the complete range of $\phi$.

\section{SUMMARY}

A numerical method is presented for calculating the transverse and longitudinal permeabilities of random arrays of cylinders. The results obtained by averaging over several configurations of the random arrays serve the useful purpose of assessing the range of validity of various approximate theories as far as their application to the estimation of the macroscopic properties of the random arrays of cylinders is concerned. It is found that the self-consistent scheme and Brinkman's approximation provide reasonable estimates of the macroscopic properties, especially considering the fact that the only information on the geometry of the two-phase 
TABLE VII. Comparison of the rigorous calculations for the longitudinal permeability with various approximate theories."

\begin{tabular}{|c|c|c|c|c|c|c|}
\hline$\phi$ & Array & Exact & $\mathrm{Br}$ & SC & $\mathbf{M B}$ & MA \\
\hline \multirow{2}{*}{0.1} & RA & $4.97 \pm 0.7$ & 5.77 & 3.5 & 7.5 & 5.4 \\
\hline & SA & 2.55 & 5.77 & 3.5 & 9.0 & 0.84 \\
\hline 0.3 & SA & 0.235 & 0.89 & 0.42 & 1.9 & 0.43 \\
\hline \multirow{2}{*}{0.5} & RA & $(6.88 \pm 1.1) 10^{-2}$ & 0.281 & 0.097 & $\cdots$ & 0.2 \\
\hline & SA & $4.45 \times 10^{-2}$ & 0.281 & 0.097 & $\cdots$ & 0.17 \\
\hline
\end{tabular}

"RA $=$ random array, $\mathrm{SA}=$ square array, $\mathrm{Br}=\mathrm{Brinkman}$ 's approximation, $\mathrm{SC}=$ self-consistent scherne, $\mathrm{MB}=$ method $\mathrm{B}$, and $\mathrm{MA}=$ method $\mathrm{A}$.

medium they require is the area fractions of the individual phases. Of the two other approximate methods considered in the present study which require, in addition to the area fractions of the two phases, the probability density function of the two-phase medium, method A which solves for the "actual" flow instead of the "disturbance" flow appears to provide better estimates of the permeabilities. It also appears that better estimates of the permeability are obtained when the "effective viscosity" of the porous medium is taken simply to be the viscosity of the fluid.

\section{APPENDIX A: A PROOF OF (14) AND (15)}

Since $u_{r}$ is a biharmonic function, it can be expressed as

$\nabla^{2} \mathbf{u}_{\mathbf{r}}=\mathbf{v}$,

$\nabla^{2} \mathbf{v}=0$.

Also since $\mathbf{u}_{\mathbf{r}}$ is regular for $r^{\prime} \equiv\left|\mathbf{r}-\mathbf{r}^{(s)}\right| \leqslant a$, it can be shown that $\mathbf{v}$ can be expressed as an infinite series of regular harmonics:

$$
\mathbf{v}=\sum_{n=0}^{\infty}\left(\mathbf{v}_{n} \cos n \theta+\tilde{\mathbf{v}}_{n} \sin n \theta\right) r^{\prime n}
$$

where

$$
\mathbf{v}_{n}=\frac{1}{n !}\left(\frac{\partial}{\partial x_{1}}\right)^{n} v\left(\mathbf{r}^{(s)}\right)=\frac{1}{n !}\left(\frac{\partial}{\partial x_{1}}\right)^{n} \nabla^{2} \mathbf{u}_{\mathbf{r}}\left(\mathbf{r}^{(s)}\right) .
$$

A similar expression applies to $\tilde{\mathbf{v}}_{n}$. Now $\mathbf{u}_{\mathrm{r}}$ can be written as a sum of the particular and homogeneous solutions of (A1) as

$$
\begin{aligned}
\mathbf{u}_{r}= & \sum_{n} \frac{1}{4(1+n)}\left(\mathbf{v}_{n} \cos n \theta+\tilde{\mathbf{v}}_{n} \sin n \theta\right) r^{\prime n+2} \\
& +\left(\mathbf{u}_{r n} \cos \theta+\tilde{\mathbf{u}}_{r n} \sin \theta\right) r^{\prime n} .
\end{aligned}
$$

Differentiating the above expression $n$ times with respect to $x_{1}$ and evaluating the result at $r^{\prime}=0$ yields

$$
\left(\frac{\partial}{\partial x_{1}}\right)^{n} \mathbf{u}_{\mathrm{r}}\left(\mathbf{r}^{(s)}\right)=n !\left(\frac{1}{4(n-1)} \mathbf{v}_{n-2}+\mathbf{u}_{r n}\right)
$$

or

$$
\mathbf{u}_{\mathrm{r} n}=\frac{1}{n !}\left[\left(\frac{\partial}{\partial x_{1}}\right)^{n}-\frac{n}{4}\left(\frac{\partial}{\partial x_{1}}\right)^{n-2} \nabla^{2}\right] \mathbf{u}_{\mathrm{r}}\left(r^{(s)}\right)
$$

on making use of (A4). Thus the coefficient of $\cos n \theta \cdot r^{\prime n}$ in (A5) is equal to

$$
\begin{aligned}
\mathbf{u}_{\mathrm{r} n}+\mathbf{v}_{n} r^{\prime 2}= & \frac{1}{n !}\left[\left(\frac{\partial}{\partial x_{1}}\right)^{n}-\frac{n}{4}\left(\frac{\partial}{\partial x_{1}}\right)^{n-2} \nabla^{2}\right. \\
& \left.+\frac{1}{4(n+1)}\left(\frac{\partial}{\partial x_{1}}\right)^{n} r^{\prime 2}\right] \mathbf{u}_{\mathbf{r}}\left(\mathbf{r}^{(s)}\right) .
\end{aligned}
$$

The above equation combined with the orthogonal properties of the trigonometric functions readily yields (14). In a similar manner the coefficients of the sine terms in (A5) yield (15).

At this point it may be appropriate to give the corresponding results for the integrals on the spherical surfaces. Thus if $\mathrm{u}$ is a biharmonic function which is regular for $r^{\prime}<a$, then

$$
\begin{aligned}
& \int_{-1}^{1} \int_{0}^{2 \pi} \mathbf{u}\left(\mathrm{r}^{\prime}\right) P_{n}^{m}(x) \cos m \theta d x d \phi \\
&= \frac{(-1)^{m} \pi 2^{n+1} r^{\prime n}}{(2 n+1)(n-m) !}\left[\left(\frac{\partial}{\partial x_{1}}\right)^{n-m} \Delta_{m}\right. \\
&-\frac{(n-m) !}{(n-2-m) !(4 n-2)}\left(\frac{\partial}{\partial x_{1}}\right)^{n-2-m} \Delta_{m} \nabla^{2} \\
&\left.+\frac{r^{2}}{(4 n+6)}\left(\frac{\partial}{\partial x_{1}}\right)^{n-m} \Delta_{m} \nabla^{2}\right] \mathbf{u}(0),
\end{aligned}
$$

where

$$
\begin{aligned}
& x_{1}=r^{\prime} \cos \theta, \quad x_{2}=r^{\prime} \sin \theta \cos \phi, \quad x_{3}=r^{\prime} \sin \theta \sin \phi, \\
& \Delta_{m}=\left(\frac{\partial}{\partial \zeta}\right)^{m}+\left(\frac{\partial}{\partial \eta}\right)^{m}, \\
& \zeta=x_{2}+i x_{3}, \quad \eta=x_{2}-i x_{3}, \quad x=\cos \theta,
\end{aligned}
$$

and $P_{n}^{m}$ is the associated Legendre function. A similar expression can also be derived for the integral containing $\sin m \phi$ instead of $\cos m \phi$ in (A9) whereby $\Delta_{m}$ would be replaced by a differential operator $\widetilde{\Delta}_{m} \equiv i\left[(\partial / \partial \xi)^{m}-(\partial /\right.$ $\left.\partial \eta)^{m}\right]$.

It should be noted that for $n=0$ and $n=1$, the above relations can be combined with the reciprocal theorem for the Stokes equation to yield Faxen's "laws" for the force and torque on the cylindrical or spherical particles placed in arbitrary flow fields at infinity. In that sense, (14), (15), and (A9) could be regarded as the generalized Faxen's "laws." Such generalized Faxen's "laws" in three dimensions have also been obtained by Mazur and van Sarloos. ${ }^{10}$ However, these authors have derived relations for the higher-order force multipoles in a Fourier space and hence their results cannot be readily compared with the results presented here.

\section{APPENDIX B: DIFFERENTATION OF $\boldsymbol{S}_{\mathbf{2}}$}

As in Part I, the lower-order derivatives of $S_{2}$ can be calculated by the termwise differentiation of (17). The formulas needed, in addition to those given in the appendix of Part I, are given below: 


$$
\begin{aligned}
& \frac{\partial^{p+q}}{\partial x_{1}^{p} \partial x_{2}^{q}}\left(r^{2} \log r\right)=(-1)^{n}(n-2) ! r^{2-n} F-2(-1)^{p+1}(n-3) ! R, \\
& F=\left\{\begin{array}{l}
(-1)^{q / 2}\{\cos n \theta-[(p-2-q) /(n-2)] \cos (n-2) \theta\}, q \text { even, } \\
(-1)^{(q+1) / 2}\{\sin n \theta-[(p-2-q) /(n-2)] \sin (n-2) \theta\}, \quad q \text { odd, }
\end{array}\right. \\
& R=\left\{\begin{array}{l}
(-1)^{q / 2} \cos (n-2) \theta, q \text { even, } \\
(-1)^{(q+1) / 2} \sin (n-2) \theta, \quad q \text { odd, }
\end{array}\right.
\end{aligned}
$$

and $n=p+q$. The above expression applies to the case $p>2$. The formulas for the cases $n<2$ or $q>2$ must be derived separately. Similarly,

$$
\begin{aligned}
& \frac{\partial^{p+q}}{\partial x_{1}^{p} \partial x_{2}^{q}}\left(r^{2+m} \cos m \theta\right) \\
& =\frac{(m+1) !}{(m-n+1) !}(-1)^{q / 2} r^{2+m-n}(\cos (m-n) \theta \\
& \left.\quad+\frac{p-q}{m-n+2} \cos (m-n+2) \theta\right)
\end{aligned}
$$

where $q$ is even. The factor $(-1)^{q / 2}$ must be replaced by $(-1)^{q+1 / 2}$ and the cosine terms by the sine terms when $q$ is odd.

The higher-order derivatives of $S_{2}$ may be evaluated by an alternate expression:

$$
\begin{gathered}
\frac{\partial^{p+q}}{\partial x_{1}^{p} \partial x_{2}^{q}} S_{2}(\mathbf{r})=-\sum_{\mathrm{r}_{\mathbf{L}}} \frac{\partial^{p+q}}{\partial x_{1}^{p} \partial x_{2}^{q}}\left(\frac{s^{2}}{2} \log s\right), \\
p+q \geqslant 5,
\end{gathered}
$$

where $\mathbf{r}_{\mathbf{L}}$ are the lattice vectors and $s=\left|\mathbf{r}-\mathbf{r}_{\mathbf{L}}\right|$.

\section{APPENDIX C: A PROOF OF (30)}

Let us begin with the definition of $U_{3}$ :

$$
\begin{aligned}
\tau U_{3}= & \int_{D_{f}} u_{3} d v=\int_{D_{f}}\left\{U_{0}+\sum_{p=1}^{N}\left[C_{\mathrm{O} 0}^{(p)} S_{1}\left(\mathbf{r}-\mathbf{R}^{(p)}\right)\right.\right. \\
& +\sum_{n=1}^{\infty} \sum_{m=0}^{1} C_{n m}^{(p)}\left(\frac{\partial}{\partial x_{1}}\right)^{n-m} \\
& \left.\left.\times\left(\frac{\partial}{\partial x_{2}}\right)^{m} S_{1}\left(\mathbf{r}-\mathbf{R}^{(p)}\right)\right]\right\} d v
\end{aligned}
$$

where $D_{f}$ is the volume occupied by the fluid within a unit cell whose volume is $\tau$. Since the integral of $S_{1}$ or any of its derivatives over the unit cell is zero, the above reduces to

$$
\begin{aligned}
\tau U_{3}= & U_{0} \tau(1-\phi)-\sum_{N=1}^{N} \int_{D_{s}^{(l)}} d v \\
& \times \sum_{p=1}^{N}\left[C_{\infty}^{(p)} S_{1}+\sum_{n=1}^{\infty} \sum_{m=0}^{1} C_{n m}^{(p)}\right. \\
& \left.\times\left(\frac{\partial}{\partial x_{1}}\right)^{n-m}\left(\frac{\partial}{\partial x_{2}}\right)^{m} S_{1}\right],
\end{aligned}
$$

where $D_{s}^{(l)}$ is the volume occupied by the $l$ th cylinder. Also from the no-slip boundary condition we have that

$$
\begin{aligned}
0= & \int_{\partial D} u_{3} d A=U_{0} \cdot 2 \pi a \\
& +\sum_{p} \int_{\partial D}\left[C_{00}^{(p)}+\sum_{n=1}^{\infty} \sum_{m=0}^{1}\left(\frac{\partial}{\partial x_{1}}\right)^{n-m}\right. \\
& \left.\times\left(\frac{\partial}{\partial x_{2}}\right)^{m} C_{n m}^{(p)}\right] S_{1} d A,
\end{aligned}
$$

where $\partial D$ is the surface of the $l$ th cylinder. Now let $\psi$ be $S_{1}$ or any derivative of $S_{1}$ and let $\psi$ be regular at $\mathbf{r}=\mathbf{R}^{(l)}$. Then a Taylor series expansion of $\psi$ near $R^{(l)}$ gives

$$
\psi=\psi_{0}+\mathbf{y} \cdot \nabla \psi_{0}+\frac{1}{2} y y: \nabla \nabla \psi_{0}+\cdots,
$$

where $\mathbf{y}=\mathbf{r}-\mathbf{R}^{(l)}$ and the subscript 0 implies the evaluation of $\psi$ or its derivatives at $\mathbf{y}=\mathbf{0}$. On integrating (C4), we obtain the following relationship between the volume and surface integrals:

$$
\frac{a}{2} \int_{\partial D^{(h)}} \psi d A-\int_{D_{s}^{(b)}} \psi d v=\frac{\pi a^{4}}{8} \nabla^{2} \psi_{0},
$$

when $\nabla^{2 m} \psi_{0} \equiv 0, m>1$. The above relation can be used to combine the volume and surface integrals in (C2) and (C3) for those terms which are regular at $\mathbf{r}=\mathbf{R}^{(l)}$. For the singular terms, the expansion of $S_{1}(r)$ near $r=0$ [cf. (16)] may be employed directly to obtain

$$
\begin{aligned}
& \frac{a}{2} \int_{\partial D(l)} S_{1}\left(\mathbf{r}-\mathbf{R}^{(l)}\right) d A-\int_{D_{s}^{(l)}} S_{1}\left(\mathbf{r}-\mathbf{R}^{(l)}\right) d v \\
& =\pi\left(-1+\frac{\pi a^{2}}{2}\right) a^{2} .
\end{aligned}
$$

Now multiplying (C3) by $a / 2$ and subtracting (C2) from it, noting that the contribution from the terms containing $C_{n m}$ $(n \neq 0)$ vanishes identically, and on using (C5), (C6), and (C1), we finally arrive at the relationship

$$
U_{3}=U_{0}-\frac{\phi}{N}\left(1-\frac{\phi}{2}\right) \sum_{1}^{N} C_{00}^{(l)}
$$

\section{APPENDIX D: DETAILS OF THE CALCULATIONS FOR THE BUYEVICH AND SHCHELCHKOVA METHOD}

Here we show how the constants $A, B$, and $B *$ are determined by solving the following two problems.

(i) Uniform flow: $u_{i}^{\infty}(I)=-\delta_{i 1}$. In this case, $\hat{u}_{i}^{\prime}=\delta_{i 1}$ at $r=1$ and we write

$$
\left\{\hat{p}^{\prime}, \hat{u}_{r}^{\prime}, \hat{u}_{\theta}^{\prime}\right\}=\left\{h_{1}, \frac{h_{2}}{r},-\frac{d h_{2}}{d r}\right\} \cos \theta,
$$

where $h_{1}$ and $h_{2}$ are functions of $r$. On substituting (D1) into the governing equations (35a) and (42) expressed in the polar coordinates together with the appropriate boundary conditions and the assumed values of $A-B^{*}$ and $B, h_{1}$ and 
$h_{2}$ can be determined. Because of the linearity of the governing equations, $\hat{\sigma}_{i j} n_{j}$ at $r=1$ must be of the form

$$
\hat{\sigma}_{i j} n_{j}=-\delta_{j 1}\left[\lambda_{1} \delta_{i j}+\lambda_{2}\left(n_{i} n_{j}-\frac{1}{2} \delta_{i j}\right)\right],
$$

where $\lambda_{1}$ and $\lambda_{2}$ are related to $h_{1}$ and $h_{2}$ at $r=1$ by

$$
2 \lambda_{1}=\frac{d^{2} h_{2}}{d r^{2}}-h_{1},-\lambda_{2}=h_{1}+\frac{d^{2} h_{2}}{d r^{2}} .
$$

On using the reciprocal theorem (51), the force on the cylinder resulting from the arbitrary flow field $\hat{u}_{i}$ can be shown to be related to $\lambda_{1}$ and $\lambda_{2}$ by

$$
\widehat{F}_{1}=\int_{\partial D} u_{i}^{\infty}\left[\lambda_{1} \delta_{i j}+\lambda_{2}\left(n_{i} n_{j}-\frac{1}{2} \delta_{i j}\right)\right] \delta_{j 1} d A,
$$

where $u_{i}^{\infty}$ must satisfy

$$
-\frac{\partial p^{\infty}}{\partial x_{i}}+\mu_{a}^{\infty} \nabla^{2} u_{i}^{\infty}-\phi B u_{i}^{\infty}=0 .
$$

To relate $\lambda_{1}$ and $\lambda_{2}$ to $B$ and $B *$, the integral in (D4) must be related to $u_{i}^{\infty}$ and $\nabla^{2} u_{i}^{\infty}$. This is done most conveniently by first writing an expression for the streamfunction $\psi^{\infty}$ satisfying (D5):

$$
\begin{aligned}
\psi^{\infty}= & \sum_{n=0}^{\infty}\left\{\left[A_{n} r^{n}+B_{n} I_{n}(\beta r)\right] \cos n \theta\right. \\
& \left.+\left[\widetilde{A}_{n} r^{n}+\widetilde{B}_{n} I_{n}(\beta r)\right] \sin n \theta\right\},
\end{aligned}
$$

where $\beta=\left(\phi B / \mu_{a}^{\infty}\right)^{1 / 2}, I_{n}$ are the modified Bessel functions, and $A_{n}, B_{n}$, etc., are the constants characterizing $u_{i}^{\infty}$. In particular, it is easy to show that

$\widetilde{B}_{1}=\frac{2}{\beta^{3}} \nabla^{2} u_{1}^{\infty}(0)$ and $\widetilde{A}_{1}=u_{1}^{\infty}(0)-\frac{1}{\beta^{2}} \nabla^{2} u_{1}^{\infty}(0)$.

Differentiating $\psi^{\infty}$ and substituting the resulting expression for $u^{\infty}$ into (D4) expressed in polar coordinates, $\widehat{F}_{1}$ can be evaluated in terms of $\widetilde{A}_{1}$ and $\widetilde{B}_{1}$. Comparing the resulting expression for $F_{1}$ obtained by adding $F_{1}^{\infty}$ and $\hat{F}_{1}$ with (46) yields the following relations:

$$
\begin{aligned}
B= & \mu_{a}^{\infty} \beta^{2}-2 \lambda_{1}, \\
B^{*}= & \mu_{a}^{\infty}\left(\frac{2}{\beta} I_{1}(\beta)-1\right) \\
& -\frac{2}{\beta^{2}}\left[\lambda_{1}\left[I_{0}(\beta)-1\right]-\lambda_{2}\left(\frac{I_{0}(\beta)}{2}-\frac{I_{1}(\beta)}{\beta}\right)\right] .
\end{aligned}
$$

Thus $\lambda_{1}$ and $\lambda_{2}$ computed from the solution of the uniform flow problem are related to $B$ and $B$ *.

(ii) Shear flow: $u_{i}^{\infty(I)}=-\left(\delta_{i 1} \delta_{j 2}+\delta_{i 2} \delta_{j 1}\right) x_{j}$. In this case we write

$$
\left\{p^{\prime}, \hat{u}_{r}^{\prime}, \hat{u}_{\theta}^{\prime}\right\}=\left\{g_{1}, \frac{g_{2}}{r}, \frac{1}{2} \frac{d g_{2}}{d r}\right\} \cos 2 \theta
$$

and determine [by solving (35a) and (42) for the assumed values of $A-B^{*}$ and $\left.B\right] \hat{\sigma}_{i j} n_{j}$ at $r=1$. The result is expressed in terms of two constants $\beta_{1}$ and $\beta_{2}$ as defined by

$$
\hat{\boldsymbol{\sigma}}^{\prime} \cdot \mathbf{n}=\beta_{1} \sin 2 \theta \mathbf{e}_{r}+\beta_{2} \cos 2 \theta \mathbf{e}_{\theta},
$$

where $e_{r}$ and $e_{\theta}$ are the unit vectors along the $r$ and $\theta$ directions, respectively, and $\beta_{1}$ and $\beta_{2}$ are related to $g_{1}$ and $g_{2}$ at $r=1$ by

$$
\beta_{1}=2-g_{1} \text { and } \beta_{2}=1+\frac{1}{2} \frac{d^{2} g_{2}}{d r^{2}} \text {. }
$$

Now, once again, by making use of the reciprocal theorem (51), the stresslet for arbitrary $\hat{u}_{i}$ can be related to $u_{i}^{\infty}, \beta_{1}$, and $\beta_{2}$. On comparing the resulting expression for the stresslet with (47), the following relations between $\beta_{1}$ and $\beta_{2}$ computed from the simple shear flow problem and $B_{1}$ and $B_{2}$ for the arbitrary flow field are obtained:

$$
\begin{aligned}
B_{1}= & \frac{1}{2}\left(\beta_{1}+\beta_{0}\right)-\frac{1}{4} \mu_{a}^{\infty}\left(8+\beta^{2}\right), \\
B_{2}= & \frac{-1}{2 \beta^{2}}\left(\beta_{1}+\beta_{2}\right)+\frac{2}{\beta^{4}}\left\{2 \beta_{1}\left(I_{0}-\frac{2 I_{1}}{\beta}\right)\right. \\
& \left.+\beta_{2}\left[\left(\beta+\frac{4}{\beta}\right) I_{1}-2 I_{0}\right]\right\}-2 u_{a}^{\infty}\left(\frac{I_{0}-1}{\beta^{2}}-\frac{1}{8}\right),
\end{aligned}
$$

where $I_{0}$ and $I_{1}$ are evaluated at $\beta$.

The numerical scheme for estimating $B$ or, equivalently, $K_{m}$ consists of the following steps.

(i) Assume $A-B^{*}$ and $B$.

(ii) Determine $\lambda_{1}, \lambda_{2}, \beta_{1}$, and $\beta_{2}$ numerically by solving for $h_{1}(r), h_{2}(r), g_{1}(r)$, and $g_{2}(r)$.

(iii) Calculate $B, B^{*}$, and $B_{2}$ from (D8), (D9), (D13), and (D14).

(iv) If the calculated values of $B$ and $A-B^{*}$ do not agree with the assumed values, then repeat steps (i)-(iii).

'A. S. Sangani and C. Yao, Phys. Fluids 31, 2426 (1988).

${ }^{2}$ H. Hasimoto, J. Fluid Mech. 5, 317 (1959).

${ }^{3}$ A. S. Sangani, Ph.D. thesis, Stanford University, 1982.

${ }^{4}$ A. S. Sangani and A. Acrivos, Int. J. Multiphase Flow 8, 193 (1982).

${ }^{5}$ E. M. Sparrow and A. L. Loeffler, AIChE J. 5, 325 (1959).

'I. D. Howells, J. Fluid Mech. 64, 449 (1974).

${ }^{7}$ Y. A. Buyevich and I. N. Schelchkova, Prog. Aerospace Sci. 18, 121 (1978).

${ }^{8}$ L. Spielman and S. L. Goren, Environ. Sci. Technol. 2, 279 (1968).

${ }^{9}$ S. Kim and W. B. Russel, J. Fluid Mech. 154, 253 (1985).

${ }^{10}$ P. Mazur and W. van Sarloos, Physica A 115, 21 (1982). 